\title{
Performance of single reshaped Judkins left catheter for transradial coronary angiography
}

\author{
Guangming Zhang, Wei Cui*, Ruiqin Xie, Fan Liu, Guoqiang Gu, Jingchao Lu, Hongmei Zheng, \\ Xiuchun Yang, Xiaohong Yang \\ Department of Cardiology, The Second Hospital of Hebei Medical University, Shijiazhuang, China \\ Email: ${ }^{*}$ cuiwei@medmail.com.cn \\ Received 21 September 2013; revised 25 October 2013; accepted 8 November 2013 \\ Copyright (C) 2013 Guangming Zhang et al. This is an open access article distributed under the Creative Commons Attribution Li- \\ cense, which permits unrestricted use, distribution, and reproduction in any medium, provided the original work is properly cited.
}

\begin{abstract}
Background: To investigate the feasibility of single reshaped Judkins left catheter for transradial coronary angiography. Methods: A total of 198 consecutive patients were enrolled in the first step study. Patients were randomly assigned to brachial type catheter group (BRACT group) or Judkins left catheter group (Judkins group). The inclusion criterion was the left coronary angiography which could be accomplished by either a brachial type catheter or a reshaped Judkins left catheter. The successful procedure was defined as both right and left coronary angiography could be completed with a single reshaped Judkins left catheter or a brachial type catheter. Furthermore, 1873 consecutive patients were continuously enrolled to observe the success rate of single Judkins left catheter for transradial coronary angiography. Results: Of the 198 patients, 191 were finally enrolled in this study according to the inclusion criteria, with 95 patients in BRACT group and 96 in Judkins group. There were no significant differences on baseline between the two groups. Procedure success rate was $84.10 \%$ with a fluoroscopic exposure time of $3.81 \pm$ $0.43 \mathrm{~min}$ in BRA-CT group, and $81.60 \%$ with a fluoroscopic exposure time of $4.05 \pm 0.48 \mathrm{~min}$ in Judkins group $(P>0.05)$. No severe complications were found in either of the two groups. The success rates of $\mathbf{1 8 6 9}$ patients with single Judkins left catheter for transradial coronary angiography were $75.33 \%$. There were no complications in those patients. Conclusions: Transradial coronary angiography with a reshaped single Judkins left catheter is feasible and practical, with an accepted procedure success rate. This method should be worthy of further clinical validation in a larger scale population.
\end{abstract}

*Corresponding author.
Keywords: Coronary Angiography; Transradial; Judkins Left Catheter

\section{INTRODUCTION}

Transradial coronary percutaneous procedures are associated with reduced entry site complications compared to transfemoral [1], and transradial procedures are becoming widely accepted by operators and patients because of the patients resuming activity immediately after the procedure and avoiding the discomfort of forced, prolonged bed rest. But one of the drawbacks for transradial procedures is related to the possibility of radial artery damage, which is mostly related to the insertion of oversized sheaths, but possibly also to catheter exchange [2]. Potential consequences of arterial injury are spasm and radial artery occlusion.

The purpose of this study was to evaluate the feasibility of single reshaped Judkins left catheter for engaging both coronary arteries via the right transradial artery approach, compared with conventional brachial type catheter BRACT.

\section{METHODS}

\subsection{Patient Selection}

From January to December 2006, A total of 198 inpatients undergoing transradial diagnostic coronary angiography or elective percutaneous coronary intervention were consecutively enrolled in the first step study. Patients were randomly assigned to Judkins left catheter group (Judkins group) or brachial type catheter BRACT group (BRACT group). The inclusion criterion was patients with a positive Allen test, and only those in whom the left coronary angiography could be accomplished with either a BRACT catheter or a reshaped Judkins left catheter were analyzed. 
In addition, from January 2006 to April 2012, included 198 inpatients in above analysis, 1873 inpatients undergoing transradial diagnostic coronary angiography or elective percutaneous coronary intervention were consecutively enrolled. All of them were underoperated with single Judkins left catheter for transradial coronary angiography. All patients provided written, informed consent before catheterization.

\subsection{Judkins Left Catheter Reshaped}

Before the Judkins left catheter was used for coronary angiography, the secondary curve of the regular catheter was reshaped to a right angle by forcefully sliding the catheter through fingers in order to obtain a configuration similar to the brachial type catheter (Figure 1).

\subsection{Catheterization Procedures}

Transradial angiography was performed just as the usual right radial approach. The radial artery was punctured with a radial artery needle, and a 0.025 "' guidewire was inserted through the needle. A 12-cm-long $6 \mathrm{~F}$ radial sheath was then inserted. Heparin was given as a bolus intravenous infusion with a dose of 3000 - 5000 IU. The sheath was removed immediately after the procedure. Hemostasis was achieved by radial compression with an artery compression bandage.

Diagnostic catheters used were either reshaped Judkins Left catheter (MERIT MEDICAL, USA) or the BRACT (MERIT MEDICAL, USA). The selection of diagnostic catheter was randomized. Judkins left 3.5 catheters were usually used, but Judkins left 4.0 catheter was also selected when ascending aorta was overwrite. When reshaped Judkins left catheters were used, after the left coronary angiography was done, the reshaped Judkins left catheter was rotated clockwise $90^{\circ}-180^{\circ}$ under fluoroscopy guidance using a LAO projection, and this maneuver usually made the catheter engage the right coronary artery easily. The skill for the BRACT catheter was the similar to that described above.

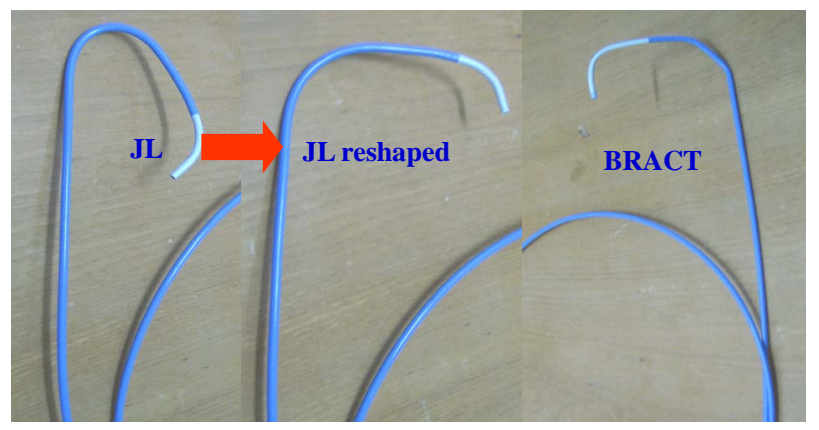

(a)

(b)

(c)

Figure 1. Show the original JL (a) is reshaped (b) to a configuration similar to BRACT catheter (c).

\subsection{Observations and Definitions}

Procedure success rate: the successful angiographic procedures were recorded in Judkins group or BRACT group. The successful procedure was defined as both right and left coronary angiography could be completed with a single reshaped Judkins left catheter or a BRACT catheter. Total fluoroscopic time: four to five conventional projections were used for left coronary angiography, and two for right coronary angiography. The total fluoroscopic time was recorded in every patient during transradial angiographic procedures. Complications: We observed the patients until 3rd day after the procedure, procedure-related complications were observed, including the need for surgical correction of vascular access site, permanent neurological deficit, radial artery occlusion, and peri-procedural Q-wave or non Q-wave myocardial infarction or death.

\subsection{Statistical Analysis}

All data were prospectively recorded on standardized forms. Data are presented as mean \pm standard deviation for continuous variables and as the percentage for categorical variables. Continuous variables were analyzed by an independent-samples $t$-test. Categorical varia bles were compared by $x^{2}$ analysis. Statistical analysis was performed with the SPSS, version 11.5, software package (SPSS, Chicago, IL). A $P$-value $<0.05$ was considered statistically significant.

\section{RESULTS}

\subsection{Baseline Data}

Of the 198 patients, 191 patients were eligible for the study, including 95 patients in BRACT group and 96 in Judkins group. The $\mathrm{JL}_{3.5}$ catheter was used in 86 patients and $\mathrm{JL}_{4.0}$ in 10 cases in Judkins group. The mean patient age was $56.84 \pm 9.50$ years, with $28.3 \%$ females. The patients in the Judkins group and BRACT group were well matched for age, sex, and other demographic data (Table 1).

Of the 1873 patients, 1869 patients were used with single reshaped Judkins left catheter for transradial coronary angiography, the mean age was $57.99 \pm 4.84$ years, $27.3 \%$ were female. All of the patients had stable hemodynamics and good radial artery pulses (positive Allen test). The study were not include of the other approach if the radial artery approach failed.

\subsection{Procedural Characteristics between Two Groups}

In the Judkins group, 78 of 96 patients were completed the procedure successfully with a single reshaped Judkins left catheter, with a procedure success rate of 
81.25\%. While 80 of 95 patients were completed the procedure with a BRACT catheter, yielding a procedure success rate of $84.21 \%$. The procedure success rate in the Judkins group showed no significant difference compared with that in the BRACT group $(P=0.70)$ (Table 2).

Total fluoroscopic time was $4.28 \pm 0.48 \mathrm{~min}$ in Judkins group, and $4.21 \pm 0.43 \mathrm{~min}$ in BRACT group. There were no significant difference in total fluoroscopic time between the two groups ( $P=0.29$ ) (Table 2).

No significantly statistical differences between the groups regarding to radial artery spasm and angiographic or clinical complications (Table 2).

\subsection{Success Rates of Single Reshaped Judkins Left Catheter}

Of the 1873 patients, 1869 patients were eligible for the study. Overall, 1408 cases (75.33\%) were performed both left and right coronary angiography with only one reshaped Judkins left catheter, and 459 cases (24.56\%) were performed with two catheters because the Judkins left catheter couldn't reach to right coronary artery

Table 1. Baseline patient's characteristics.

\begin{tabular}{cccc}
\hline & $\begin{array}{c}\text { Judkins group } \\
(\mathrm{n}=96)\end{array}$ & $\begin{array}{c}\text { BRACT group } \\
(\mathrm{n}=95)\end{array}$ & $P$-values \\
\hline Age (years) & $56.0 \pm 9.9$ & $57.1 \pm 8.9$ & 0.17 \\
Male gender (n (\%)) & $68(70.8)$ & $73(76.8)$ & 0.06 \\
Hemoglobin (g/l) & $130.1 \pm 11.2$ & $135.3 \pm 12.6$ & 0.16 \\
BMI (kg/m²) & $25.3 \pm 3.59$ & $25.7 \pm 3.10$ & 0.08 \\
Risk factors (n (\%)) & & & \\
Hypertension & $64(66.7)$ & $66(69.5)$ & 0.36 \\
Hyperlipidemia & $29(30.2)$ & $33(34.7)$ & 0.26 \\
Diabetes mellitus & $20(20.8)$ & $22(23.2)$ & 0.33 \\
Smoking & $52(54.2)$ & $52(54.7)$ & 0.97 \\
\hline
\end{tabular}

Values are expressed as mean $\pm \mathrm{SD}$ or $\mathrm{n}(\%)$. $P$-values derived from independent sample $t$-test for continuous variables or $x^{2}$ test for categorical variables. BMI: body mass index.

Table 2. Safety and efficacy between Judkins group and BRACT group.

\begin{tabular}{cccc}
\hline & $\begin{array}{c}\text { Judkins group } \\
(\mathrm{n}=96)\end{array}$ & $\begin{array}{c}\text { BRACT group } \\
(\mathrm{n}=95)\end{array}$ & P values \\
\hline Success rates (n (\%)) & $78(81.25)$ & $80(84.21)$ & 0.70 \\
Fluoroscopic time (min) & $4.28 \pm 0.48$ & $4.21 \pm 0.43$ & 0.29 \\
Complications (n (\%)) & $0(0)$ & $0(0)$ & 1.00 \\
\hline
\end{tabular}

Values are expressed as mean $\pm \mathrm{SD}$ or $\mathrm{n}(\%)$. $P$-values derived from independent sample $t$-test for continuous variables or $x^{2}$ test for categorical variables. ostium. The other 2 cases had abnormal right coronary artery ostium that the Judkins right catheter couldn't reach to right coronary artery ostium, so we had to use the Amplatz Right (AR) catheter to reach to right coronary artery ostium.

\section{DISCUSSION}

In recent years, the transradial approach has been used increasingly as an alternative to femoral approach for percutaneous coronary diagnosis and intervention. Most of the diagnostic catheters that are currently used for transradial coronary angiography are the Judkins, Amplatz, which have curves originally designed for a femoral approach. The technical and anatomic aspects of transradial access are different from those of femoral access, thus making manipulation of conventional diagnostic catheters sometimes difficult and time-consuming. Therefore, many attempts have been made to design a catheter for a transradial approach for routine diagnostic and interventional coronary procedures, trying to engage both coronary arteries by one single catheter [3]. For example, the 5F Tiger II (Terumo Corporation, Tokyo, Japan) catheters were tested to prove the feasibility, safety, and performance as a kind of multipurpose catheter for transradial coronary angiography [4]. The Brachial Type K (Terumo Outlook, Leuven, Belgium) catheters were also proved to to perform both left and right coronary angiography with a single catheter [5].

The diagnostic catheter that currently used for transradial coronary angiography in most hospitals in China is the multipurpose brachial type catheter (BRACT). BRACT catheter is more costly than Judkins, which limits the useage of BRACT catheter to some extent. This study demonstrated the feasibility and safety of single reshaped Judkins left catheter for engaging both coronary arteries via the right transradial artery approach.

The present study showed that by using a reshaped Judkins left catheter, it was possible to successfully perform both left and right coronary angiography in 75.33\% of the cases, which was very similar to conventional radial catheter with respect to procedure success rate and fluoroscopic time. Moreover, there were no instances of aortic or coronary dissection. The manipulation of diagnostic catheters also did not lead to excessive spasm. According to our experience, the 5F Judkins left catheter was more suitable for reshaping, although the 6F catheter can also be reshaped. Another advantage by using a reshaped Judkins left catheter was that this reshaped catheter didn't need a guidewire support for engaging either the right or the left coronary arteries, even when the initial attempt for engaging failed. This will simplify the manipulation and reduce the potential complications related to guidewire exchange. 


\section{STUDY LIMITATIONS}

This study is limited by its small sample size. Otherwise, we didn’t compare reshaped left Judkins catheter with other catheter designs, including non-reshaped left Judkins catheters, and await confirmation by prospective analyses.

\section{CONCLUSION}

Transradial coronary angiography with a reshaped single Judkins left catheter is feasible and practical, with an accepted procedure success rate. This method should be worthy of further clinical validation in a lager scale population.

\section{REFERENCES}

[1] Louvard, Y., Lefevre, T., Allain, A., et al. (2001) Coronary angiography through the radial or the femoral approach: The CARAFE study. Catheterization and Cardiovascular Interventions, 52, 181-187. http://dx.doi.org/10.1002/1522-726X(200102)52:2<181::

\section{AID-CCD1044>3.0.CO;2-G}

[2] Saito, S., Ikei, H., Hosokawa, G., et al. (1999) Influence of the ratio between radial artery inner diameter and sheath outer diameter on radial artery flow after transradial coronary intervention. Catheterization and Cardiovascular Interventions, 46, 173-178.

http://dx.doi.org/10.1002/(SICI)1522-726X(199902)46:2 $\leq 173::$ AID-CCD12>3.0.CO;2-4

[3] Ootomo, T., Meguro, T., Endoh, N., et al. (2002) A new miniature catheter with side-holes for percutaneous transradial or transbrachial coronary angiography. Journal of Invasive Cardiology, 14, 379-384.

[4] Kim, S.M., Kim, D.K., Kim, D.I., et al. (2006) Novel diagnostic catheter specifically designed for both coronary arteries via the right transradial approach. A prospective, randomized trial of Tiger II vs. Judkins catheters. The International Journal of Cardiovascular Imaging, 22, 295-303. http://dx.doi.org/10.1007/s10554-005-9029-8

[5] Sanmartin, M., Esparza, J., Moxica, J., et al. (2005) Safety and efficacy of a multipurpose coronary angiography strategy using the transradial technique. Journal of Invasive Cardiology, 17, 594-597. 